\title{
The genus Morinda (Rubiaceae) in Thailand
}

\author{
Wirot Kesonbuaa ${ }^{\mathrm{a}}$, Pranom Chantaranothai ${ }^{\mathrm{b}, *}$ \\ ${ }^{a}$ Biological Science Department, Faculty of Science, Ubon Ratchathani University, \\ Ubon Ratchathani 34190 Thailand \\ b Applied Taxonomic Research Centre, Department of Biology, Faculty of Science, Khon Kaen University, \\ Khon Kaen 40002 Thailand
}

${ }^{*}$ Corresponding author, e-mail: pranom@kku.ac.th

Received 13 Nov 2012

Accepted 23 May 2013

\begin{abstract}
The genus Morinda (Rubiaceae) is revised and presented with a key to species, descriptions with distributional and ecological data, together with illustrations of some species. Nine species are considered, one of which is divided into two varieties. Two species and one variety are endemic to the country. M. talmyi is typified. M. longifolia and M. pumila are reduced to synonymy with $M$. pandurifolia and $M$. tomentosa, respectively.
\end{abstract}

KEYWORDS: revision, endemic, distributional data, taxonomic studies

\section{INTRODUCTION}

The genus Morinda was described by Linnaeus ${ }^{1}$, including three species, M. citrifolia L., M. royoc L., and M. umbellata $\mathrm{L}$. The genus comprises ca. 40 species, distributed mainly in the pantropical region ${ }^{2}$. The species occur in primary and secondary forests and scrubs in the lowlands as well as at higher altitudes. These plants are distinguished from other genera of the Rubiaceae by the following characters: the growth habits are small trees or shrubs, rarely lianas. Leaves are petiolate or subsessile, with chartaceous to coriaceous. The inflorescences are either leaf-opposed or axillary, with a stalked head of flowers which are fused together by their ovaries. Flowers are bisexual; stamens inserted in the tube or near the throat; the ovary is bilocular, with longitudinal false dissepiments, each carpel has 2 ovules; style with 2-lobed stigma. The fruits are fused together as syncarps; seeds are globose or semi-globose ${ }^{2-4}$. The genus Morinda is placed in the tribe Morindeae, subfamily Rubioideae and the systematic affinities of the genus have been discussed by Baillon ${ }^{5}$, Vercourt ${ }^{6}$, Bremekamp ${ }^{7}$, Johansson ${ }^{8,9}$, Robbrecht ${ }^{10}$, and Razafimandimbison et al ${ }^{2,3,11,12}$.

Taxonomic studies of the genus Morinda in Thailand have been presented on checklist or description of new species. Craib ${ }^{13}$ described five new species from Thailand, viz., M. cinnamomea, M. longifolia, M. nana, M. pumila, and M. scabrida. He also published the list of the species from Thailand ${ }^{14}$. Fifteen species and three varieties were recorded but no key made available.

The lack of a revision has led to difficulty in identification of the Thai species and has frequently given rise to wrong determinations. Hence this present work is considered worthwhile undertaking an up-todate study. Nine species (10 taxa) are now recognized from Thailand.

\section{TAXONOMIC TREATMENT}

\section{Morinda}

L., Sp. Pl.: 176. 1753; Benth. \& F. Muell., Fl. Austral. 3: 423. 1866; Kurz, Forest Fl. Burma 2: 58. 1877; Hook. f., Fl. Brit. Ind. 3: 157. 1880; Pitard in Lecomte, Fl. Indo-Chine 3: 418. 1922; Ridl., Fl. Mal. Pen. 2: 117. 1923; Backer \& Bakh. f., Fl. Java 2: 349. 1965; Ohwi, Fl. Jap.: 826. 1965; Wong, Malay Nat. J. 38: 95.1984 \& in Ng, Tree Fl. Mal. 4: 376. 1989.

Trees, shrubs or scandent shrubs; young branches terete or subterete, with a longitudinal groove in the middle on the wider side, bark smooth, glabrous or with sparse and short hairs; older branches subterete, bark smooth or shallowly fissured, glabrous or with sparse and short hairs. Leaves simple, opposite; blade variable in shape, elliptic, broadly elliptic, oblong, obovate, lanceolate or fiddle-like shaped; base attenuate, cuneate to rounded; apex acute or acuminate; coriaceous or membranous, glabrous, with sparse and short hairs, puberulous or tomentose; lateral veins prominent or obscure, domatia usually present in axils of lateral veins on the lower leaf surface. Interpetiolar stipules acute to acuminate, truncate, obtuse, bilobed or foliaceous, usually partially connate and forming a sheath enclosing basal parts of the internode. Leaf-opposed inflorescences: solitary or paired, with ovaries connate into pedunculate capitula, each capitulum composes of many flowers fused by their 
Table 1 Key to species of Morinda in Thailand.

1. Trees or shrubs. Leaves very uniform. Pedunculate capitula at anthesis more than $4 \mathrm{~mm}$ long

2. Trees. Corolla hypocrateriform, lobes oblong, corolla tube up to $1.8 \mathrm{~cm}$ long

3. Leaves less than $14 \mathrm{~cm}$ wide, venation prominent. Infructescences globose, subglobose or oblique, ripening black

4. Leaves margin entire. Corolla tube more than $1 \mathrm{~cm}$ long. Filaments straight

5. Leaves and flowers glabrous

5. Leaves and flowers hairy

4. Leaves margin undulate. Corolla tube up to $1 \mathrm{~cm}$ long. Filaments curved

3. M. coreia

9. M. tomentosa

4. M. elliptica

3. Leaves more than $14 \mathrm{~cm}$ wide, venation obscure. Infructescences ellipsoid, ripening whitish yellow or whitish green

2. M. citrifolia

2. Shrubs. Corolla infundibular, lobes ovate or elliptic, corolla tube more than $1.8 \mathrm{~cm}$ long

6. Both surfaces of leaf glabrous or with sparse and short hairs. Fruitlets free, or slightly connate at the base

1. M. angustifolia

6. Upper surface of leaf with sparse and short hairs, lower one with dense hairs. Fruitlets fused 7. M. scabrida

1. Shrubs or scandent shrubs. Leaves variable in shape. Pedunculate capitula at anthesis sessile or up to $4 \mathrm{~mm}$ long

7. Leaves coriaceous, with sparse and short hairs on both surfaces, upper leaf surface dark green, venation prominent

8. Leaves $0.5-1 \times 1.5-2 \mathrm{~cm}$, lateral veins $4-5$ pairs

8. Leaves $1-3.5 \times 3-15 \mathrm{~cm}$, lateral veins $8-12$ pairs

5. M. nana

7. Leaves membranaceous, glabrous on both surfaces, upper leaf surface light green venation obscure

6. M. pandurifolia

8. M. talmyi

ovaries. Flowers (4-)5-(6-) merous, fragrant, bisexual; usually heterostylous, with brevistylous and longistylous flowers; calyx tube at least basally connate; calyx lobes truncate or denticulate, acute or obtuse, one teeth sometimes enlarged and subfoliaceous; corolla infundibular or hypocrateriform, white or coloured; corolla tube inside glabrous or usually covered with sparse and short hairs and in upper half villous or with long hairs, lower part of tube with or without longitudinal narrow slits below stamens; corolla lobes oblong or elliptic, glabrous or usually covered with sparse and short hairs at base. Stamens included or exserted in the corolla tube; filaments short or long, anthers included or exserted. Ovary 2-locular, with 2 ovules per locule; stigma bilobed, included or slightly exserted. Infructescences globose, subglobose, ellipsoid or oblique, consist of fused drupes or fruitlets not distinctly fused. Seed 1-4 per fruitlet, flattened, concavo-convex, ovoid or ellipsoid, with a narrow transversal slit at lower part, seed coat membranous.

About 40 species, the majority widely distributed throughout tropical and subtropical regions, nine species in Thailand (Table 1).

1. Morinda angustifolia Roxb., [Hort. Beng.: 15. 1814, nom nud.] Cor. Pl. 3: 32, t. 237. 1819 \& Fl. Ind. 2: 201. 1824; DC., Prodr. 4: 447. 1830; Kurz, Forest Fl. Burma 2: 61. 1877; Hook. f., Fl. Brit. Ind. 3: 156. 1880; Brand., Ind. Trees: 392. 1906; Pitard in Lecomte, Fl. Indo-Chine 3: 419. 1922; Craib, Fl. Siam. Enum. 2: 174. 1934; Hara, Fl. E. Himal.: 312.
1966. Type: India, Silhet, 12 July 1809, Wallich, Cat no. 8420 (holotype K-W!).

\section{Key to the varieties}
1. Leaves glabrous
1. Leaves hairy
a. var. angustifolia
b. var. scabridula

a. var. angustifolia Shrubs up to $3 \mathrm{~m}$; young branches subterete, bark smooth, greenish, glabrous; older branches subterete, bark smooth or shallowly fissured, brownish to grey. Leaves elliptic, oblong or obovate, 3-7 by 19-25 cm, coriaceous; base attenuate or cuneate; margin entire; apex acuminate; upper leaf surface light green, lower leaf surface greenish, glabrous on both surfaces; lateral veins 7-9 pairs, venation prominent; drying dark brown; petiole 0.5$1 \mathrm{~cm}$ long, glabrous. Stipules triangular, acuminate, connate at base, glabrous, usually persistent. Leafopposed inflorescence: capitula solitary or paired; peduncle at anthesis 1-3 cm long. Flowers 4-5 (-6)merous, fragrant, bisexual, heterostylous; calyx tube at least basally connate; lobes truncate or obtuse; corolla infundibular, greenish or white to pink; corolla tube 2-2.7 cm long, inside glabrous, outside with sparse and short hairs; corolla lobes ovate or elliptic, $3.8-7$ by $10-15 \mathrm{~mm}$. Stamens included in the corolla tube, attached to the tube at the middle or lower part; filaments linear, 0.5-1 mm long; anthers yellow, oblong, 4.5-5.5 mm long; style white, 5-8 mm long in brevistylous flower, 18-27 mm long in longistylous flower, glabrous. Ovary 2-locular; stigma bilobed, included or slightly exserted from the corolla throat, 


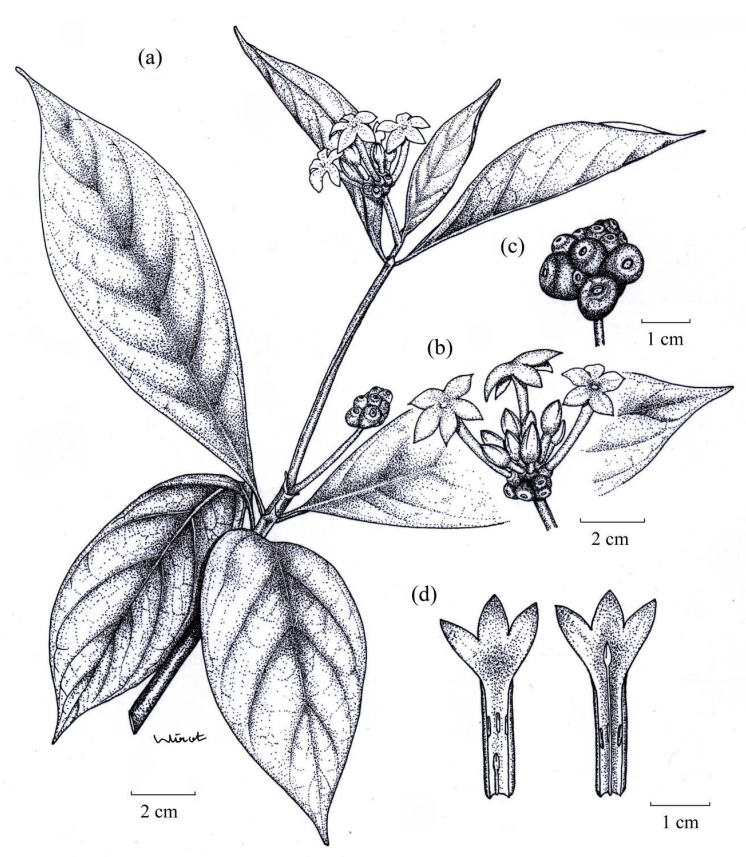

Fig. 1 M. angustifolia Roxb. var. scabridula Craib: (a) habit; (b) inflorescence; (c) infructescence; (d) longitudinal section of brevistylous and longistylous flowers. (a), (b) and (c) from W. Kesonbua 87; (d) from W. Kesonbua 87 (brevistylous flower) and W. Kesonbua 88 (longistylous flower). Drawn by W. Kesonbua.

3-8 mm long. Infructescences oblique, with 16-37 fruitlets; fruitlet free, individual not distinctly fused. Seed ovoid or ellipsoid, $2-3$ by $5-6 \mathrm{~mm}$, testa rough.

Thailand.- NORTHERN: Chiang Mai [Mae Rim district, 18 Mar. 1994, H.B.G. Garrett 171 (ABD 2 sheets, BKF, BM, K, L, TCD)]; Lampang [Mae Tha district, 26 Oct. 1983, Anderson 5237 (BKF)].

Distribution.- India, Bangladesh, Bhutan, Myanmar, Indo-China.

Ecology.- Scattered in dry dipterocarp and mixed deciduous forests, alt. 600-1000 m. Flowering period: March-October.

Vernacular.- 'Salak ban' (Northern).

Note.-M. angustifolia is distinguished from all other Thai species of Morinda in its fruits free or less connate at the base. The corolla varies in colour from white to pink.

b. var. scabridula Craib, Fl. Siam. Enum. 2: 174. 1934. Type: Thailand, Phitsanulok, Nakhon Thai district, A.F.G. Kerr 8907 (holotype BK!; isotypes ABD!, BM!, K!). Fig. 1.

Leaves covered with sparse and short hairs throughout.
Thailand.- NORTHERN: Mae Hong Son [Mae Sariang, 18 Sept. 1967, Iwatsuki \& Fukuoka T-10370 (BKF, P)]; Chiang Mai [Ban Bo Luang, 12 June 1973, Geesink, Phanichapol \& Santisuk 5850 (AAU); Doi Chiang Dao, 13 July 1998, Chatchai 894 (BKF), 1 April 1955, Garrett 1446 (K, P); Doi Suthep, 23 Mar. 1965, Chermsirivathana 318 (BKF) \& 356 (BK), 23 April 1991, Dawson 26 (BK, K) \& 27 (BKF, K), 23 Feb. 1988, Maxwell 88-223 (BKF, CMU, L), 1 April 1988, Maxwell 88-417 (CMU), 29 Jan. 1989, Maxwell 89-107 (CMU), 24 Mar. 1992, Soradat 425 (BKF); Mae Rim district, 18 Mar. 1994, BGO. Staff 432 (QBG), 5 Feb. 2001, Pongamornkul 613 (QBG); Mae Sa Botanic Garden, 17 April 1964, Pooma 411 (BKF)]; Chiang Rai [Mae Salong, 27 Feb. 1926, Winit 1610 (BK, K)]; Nan [NE of Santisuk, km 4555 along road 1081, Larsen, Larsen, Niyomdham, Sirirugsa, Tarvengadum \& Nrgaard 43537 (AAU, BKF, PSU, P)]; Lampang [Jae Son National Park, 14 Feb. 1996, Maxwell 96-208 (BKF, L)]; Phitsanulok [Nakhon Thai district, Kerr 8907 (ABD, BK, BM, K); Thung Salang Luang National Park, 19 Feb. 1964, Kerr 4736 (BK, BM, K), 7 April 1964, Pradit 785 (BK), 19 April 1964, Pradit 813 (BK); Phu Soi Dao National Park, 9 Mar. 2003, Kesonbua 87 (KKU) \& 88 (KKU)]; NORTH-EASTERN: Loei [Dan Sai, 18 April 1968, Chermsirivathana 949 (BK)]; SOUTHWESTERN: Kanchanaburi [Si Sawat, 24 May 1962, Kasem 169 (BK)].

Distribution.-Endemic to Thailand.

Ecology.- In deciduous dipterocarp, dry evergreen, hill evergreen and mixed deciduous forests, alt. $120-1200 \mathrm{~m}$. Flowering period: January-May.

Vernacular.— 'Charak dong', 'tueng sai', 'salak', 'yo din' (Chiang Mai); 'salak pa' (Lampang).

Note.- Var. scabridula differs from the typical variety only in its leaves having short hairs on both surfaces.

2. Morinda citrifolia L., Sp. Pl.: 176. 1753; Roxb., Fl. Ind. ed. 2, 2: 196. 1824; Miq., Fl. Ind. Bat. 2: 242. 1856; Benth. \& F. Muell., Fl. Austral. 3: 423. 1866; Kurz, Forest Fl. Burma 2: 60. 1877; Hook. f., Fl. Brit. Ind. 3: 155. 1880; Brand., Ind. Trees: 392. 1906; Pitard in Lecomte, Fl. Indo-Chine 3: 423. 1922; Ridl., Fl. Mal. Pen. 2: 117. 1923; Craib, Fl. Siam. Enum. 2: 175. 1934; Backer \& Bakh. f., Fl. Java. 2: 351. 1965; Wong, Malay Nat. J. 38: 94.1984 \& in Ng, Tree Fl. Mal. 4: 377. 1989.—M. bracteata Roxb., Fl. Ind. 2: 198. 1824; Thw., Enum. Pl. Zeyl.: 144. 1859. Type: India, Hermann s.n. (lectotype BM!).

Trees up to $10 \mathrm{~m}$; young branches terete, bark greenish or grey, glabrous; older branches subterete, 
bark smooth, grey to light brownish grey. Leaves elliptic or broadly elliptic, $14.5-25$ by $15.5-35.5 \mathrm{~cm}$, coriaceous; base attenuate or cuneate; margin entire or less undulate; apex acute; upper leaf surface glossy dark green, lower leaf surface pale green, glabrous on both surfaces; lateral veins 5-7 pairs, venation obscure, domatia usually presence in axils of lateral veins on the lower surface; drying dark brown or black; petiole 1-2.5 (-3.8) cm long, glabrous. Stipules truncate or obtuse to acuminate or bilobed, sometimes almost foliaceous, connate at base, usually persistent. Leaf-opposed inflorescence: capitula solitary peduncle at anthesis 5-15 mm long. Flowers 5-6merous, fragrant, bisexual; calyx tube basally connate; calyx lobes truncate, one teeth sometimes enlarged and subfoliaceous; corolla hypocrateriform, white or greenish; corolla tube $8-10 \mathrm{~mm}$ long, with long hairs or villose in upper $1 / 3$ and usually in tube, outside white, glabrous; corolla lobes oblong, $1.5-2.5$ by 4 $7 \mathrm{~mm}$, inside white, glabrescent, outside glabrous. Stamens included in the corolla tube or immediately below the corolla throat; filaments $2-3 \mathrm{~mm}$ long; anthers yellow, oblong, 2.5-3 mm long; style white, 8-10 mm long, glabrous. Ovary 2-locular; stigma greenish, bilobed, $1.5-3 \mathrm{~mm}$ long. Infructescences ellipsoid, with 50-90 fruitlets, glabrous; ripening whitish yellow or whitish green. Seed concavo-convex or ovoid, $4-5$ by $8-11 \mathrm{~mm}$, with a slit at lower part, testa rough.

Thailand.- Widely cultivated in all provinces; NORTHERN: Chiang Mai [Muang district, 25 April 1991, Maxwell 91-372 (CMU, P); Phrao district, 7 Dec. 1997, Watthana 54 (QBG)]; NORTHEASTERN: Khon Kaen [Khon Kaen University, 4 June 2002, Kesonbua 44 (KKU)]; EASTERN: Nakhon Ratchasima [Khao Yai National Park, 16 April 1927, Kerr 12767 (ABD, BKF, BM, K)]; CENTRAL: Pathum Thani [not located, 30 July 1973, Murata \& Fukuoka T-17326 (BKF); Samut Prakan [Pak Nam, 26 April 1931, Kerr 20446 (ABD, BKF, BM, K, TCD), 25 April 1920, Marcan 239 (AAU, BM)]; SOUTH-EASTERN: Trat [Ko Chang, 2 May 1974, Geesink, Hattink \& Phengklai 6606 (BKF, P), 4 April 1923, Marcan 1293 (AAU, ABD 2 sheets, BM)]; PENINSULAR: Surat Thani [Ko Tao, 20 July 1927, Kerr 12977 (ABD, BK, BKF, BM, K, E, TCD)]; Satun [Tarutao Island, 1 Dec. 1979, Congdon 209 (AAU, PSU), 1 May 2003, Kesonbua 76 (KKU); Adang Island, 15 Jan. 1928, Kerr 14086 (ABD, BKF, BM, K, TCD)]; Songkhla [not located, 10 Sept. 1979, Choatip 24 (PSU); Ko Kobe, Songkhla lake, 26 May 1984, Sirirugsa 838 (PSU)].

Distribution.- Widely cultivated throughout the tropics.

Ecology.- Cultivation. Flowering period: all year round.

Vernacular.- 'Mata suea' (Northern); 'yo', 'yo ban' (Central).

Note.- M. citrifolia is distinct from all other Morinda in having enlarged and leaf-like stipules, ellipsoid fruits and leaves larger than other species. Some specimens have one enlarged and subfoliaceous calyx lobe.

3. Morinda coreia Ham., Trans. Linn. Soc. London 13: 537. 1822. Type: India, Malabar, Mithila, Sukanagor, B. Hamilton 709 (holotype: E; isotype K!).- M. tinctoria Roxb., [Hort Beng.: 15. 1814, nom. nud.] Fl. Ind. 1: 197. 1824; Hook. f., Fl. Brit. Ind. 3: 156. 1880; Pitard in Lecomte, Fl. Indo-Chine 3: 424. 1922. Type: India, Roxburgh drawing 918 (lectotype K!).-M. exserta Roxb., Fl. Ind. 2: 199. 1824. Type: India, Roxburgh s.n., not seen.

Trees up to $15 \mathrm{~m}$; young branches terete to subterete, bark smooth, greenish, glabrous; older branch subterete, bark shallowly fissured, brownish to grey. Leaves elliptic, $8.5-11.5$ by $18-25.5 \mathrm{~cm}$, coriaceous; base cuneate; margin entire; apex acute or acuminate; upper leaf surface light green, lower leaf surface greenish, glabrous on both surfaces; lateral veins $7-10$ pairs, venation prominent, domatia usually presence in axils of lateral veins on the lower surface; drying dark black; petiole $1.2-3.5 \mathrm{~cm}$ long, glabrous. Stipules triangular, acute to acuminate, connate at base, usually caducous. Leaf-opposed inflorescence: capitula solitary or paired; peduncle at anthesis 2.5$6 \mathrm{~cm}$ long. Flowers 5-6-merous, fragrant, bisexual; heterostylous; calyx tube basally connate, glabrous; calyx lobes truncate or denticulate; corolla hypocrateriform, white or greenish; corolla lobes oblong, $2.5-5$ by $10-15 \mathrm{~mm}$; corolla tube $1.2-1.7 \mathrm{~cm}$ long, glabrous on both sides. Stamens included the tube or exserted in corolla tube; filaments linear, straight, 1-5 mm long; anthers yellow, oblong, 6-7 mm long; style white, glabrous, 5-7 mm long in brevistylous flower, 13-16 mm long in longistylous flower. Ovary 2-locular; stigma bilobed, 4-7 mm long, included or exserted in the corolla tube. Infructescences globose or subglobose, with 10-35 fruitlets, glabrous, ripening black. Seed ellipsoid or ovoid, $3.5-5$ by $6-9.5 \mathrm{~mm}$, testa rough.

Thailand.- NORTHERN: Mae Hong Son [Mae Sarieng, 12 July 1968, Larsen, Santisuk \& Warncke 2373 (AAU, P), 5 July 1996, Sangkhachand 347 (BK)]; Chiang Mai [Bo Luang, 12 June 1973, Geesink, Phanichapol \& Santisuk 5850 (P), 14 
June 1968, van Beusekom \& Phengklai 1236 (AAU, BK, BKF, K, L, P); Doi Inthanon, 18 July 1988, Fukuoka T-62048 (BK, BKF), 18 July 1998, Phengklai, Tamura, Niyomham \& Sangkhachand 4276 (BK, BKF, K), 24 Nov. 1969, Worawat 25 (BKF); Ob Luang, 20 April 1983, Umpai 481 (BK)]; NORTHEASTERN: Loei [Phu Kradueng, 27 Aug. 1988, Fukuoka T-63680 (BK, BKF, L); Wang Sapung, 21 May 1951, Pan 105 (BK, BKF)]; Khon Kaen [Khon Kaen University, 16 Mar. 2002, Kesonbua 7 (KKU), 26 Mar. 2002, Kesonbua 9 (KKU), 5 June 2002, Kesonbua 24 (KKU); Chum Phae, 11 Aug. 1972, Larsen, Nielsen \& Santisuk 31666 (P)]; Nakhon Ratchasima [Sakae Rat, 6 July 1991, Eidthang W-232 (BK, BKF)]; SOUTH-WESTERN: Kanchanaburi [Sai Yok, 15 July 1946, Kostermans 1166 (P), 29 Mar. 1992, Preecha 385 (BK, BKF)]; CENTRAL: Saraburi [Sam Lan forest, 13 April 1974, Maxwell 74-262 (BK, L), 7 June 1975, Maxwell 75578 (AAU, BK, L)]; SOUTH-EASTERN: Chon Buri [Toong Brong, 4 May 1971, Maxwell 71-388 (BK), 6 May 1972, Maxwell 72-211 (AAU, BK); Sichang Island, 8 May 1993, Maxwell 93-396 (BK, BKF, L, Herb., Biology, Chiang Mai University)].

Distribution. - India, Indo-China.

Ecology.- In deciduous dipterocarp, hill evergreen and mixed deciduous forests, alt. $50-1300 \mathrm{~m}$. Flowering period: February-July.

Vernacular-_ 'Khu' (Karen - Kanchanaburi); 'kho' (Karen); 'yo pa' (General).

Note.- M. coreia is easily distinguished by having tree-like habit, glabrous and coriaceous leaves, white and glabrous corolla, and the stamens which are inserted or included in the corolla tube.

4. Morinda elliptica (Hook. f.) Ridl., Jour. Str. Br. Roy. As. Soc. 79: 86. 1918 \& Fl. Mal. Pen. 2: 118. 1923; Wong, Malay Nat. J. 38: 94. 1984 \& in Ng, Tree Fl. Mal. 4: 377. 1989._- M. citrifolia var. elliptica Hook. f., Fl. Br. Ind. 3: 156. 1880; King \& Gamble, J. As. Soc. Beng. 73: 87. 1904. Type: Myanmar, Tavoy, F. de Silva \& W. Gomez in Wallich, Cat. no. 8434 (holotype K-W!).

Trees up to $10 \mathrm{~m}$; young branches subterete, bark smooth, greenish, glabrous; older branches terete, bark shallowly fissured, brownish to grey. Leaves elliptic, $4-6.5$ by $10-19.5 \mathrm{~cm}$, coriaceous; base cuneate; margin undulate; apex acute or acuminate; upper leaf surface light green, lower leaf surface greenish, glabrous on both surfaces; lateral veins 6-8 pairs, venation prominent, domatia usually presence in axils of lateral veins on the lower surface; drying dark black; petiole 1-2.5 cm long, glabrous. Stipules trian- gular, acute to acuminate, connate at base, usually caducous. Leaf-opposed inflorescence: capitula solitary or paired; peduncle at anthesis 3-6 cm long. Flowers 5 (-6)-merous, fragrant, bisexual; calyx tube at least basally connate, glabrous; calyx lobes obtuse or triangular; corolla hypocrateriform, white or greenish; corolla tube $0.6-1 \mathrm{~cm}$ long, glabrous on both sides; corolla lobes oblong, $2-2.5$ by $5.5-8 \mathrm{~mm}$, glabrous on both sides. Stamens exserted, attached to the tube by a short filament; filaments linear, curved, 1-2 mm long; anthers white or yellowish grey, oblong, 5-6 mm long; style white, 1.3-1.6 cm long, glabrous. Ovary 2locular; stigma bilobed, 4-6 mm long, exserted in the corolla tube. Infructescences subglobose or oblique, with 38-60 fruitlets, glabrous, ripening black. Seed ellipsoid or ovoid, $4.5-6$ by $6.5-8 \mathrm{~mm}$, testa rough

Thailand.- PENINSULAR: Ranong [Kapor district, 13 Aug. 1977, Santisuk 1240 (PSU); Ngaw waterfall, 27 April 1974, Tippan 127 (BK)]; Phangnga [Khong Nang Yon, 28 April 1973, Geesink \& Santisuk 4981 (AAU, E, K, L, P)]; Nakhon Si Thammarat [Ban Kiri Wong, 26 April 2003, Kesonbua 74 (KKU)]; Satun [Adang Island, 6 Dec. 1979, Congdon 225 (AAU, PSU)]; Phuket [Khao Chiang Khrod and Khao Sa, 7 May 1968, van Beusekom \& Phengklai 630 (P)]; Krabi [Klong Chilad, 13 July 1992, Larsen, Larsen, Niyomdham, Sirirugsa, Tarvengadum \& Nrgaard $43361 \& 43363(\mathbf{P}), 15 \mathrm{~km}$ NW of Krabi, 15 July 1992, Larsen, Larsen, Niyomdham, Sirirugsa, Tarvengadum \& Nrgaard 43421 (P)]; Songkhla [Had Yai district, 23 April 1985, Vacharee 630 (BK); Khao Kor Hong, 3 Jan. 1974, JRC PS 218 (PSU), 8 Nov. 1990, Larsen, Larsen, Barfod, Nanakorn, Ueachirakan \& Sirirugsa 41223 (BKF), 2 July 1993, Puff \& Sridith 930 702-1/2 (AAU, PSU), 26 Aug. 1974, Sutus 33 (PSU); Klong Hoy Kong, 6 May 1985, Tongseedam 22 (PSU, L)]; Yala [Betong district, 13 Aug. 1923, Kerr 7637 (AAU, ABD, BK, BM, E, K); Nikom Kua Long, 1 Dec. 1972, Pinnin et al. 1 (P)]; Narathiwat [Khao Samnak, 23 April 1986, Niyomdham et al. 1210 (AAU, BKF, P); Bacho district, 22 April 1961, Sangkhachand 77 (L, K, P), 16 Dec. 1968, Sangkhachand 1573 (BK); Tak Bai, 9 Sept. 1987, Sangkhachand \& Sriboonma, 1567 (BKF, P)].

Distribution.- India, Malaysia.

Ecology.- In evergreen rainforest and mixed deciduous forests, alt. 0-200 m. Flowering period: January-August.

Vernacular-_ 'Ka-mu-du' (Malay-Narathiwat); 'yo pa' (Trang, Satun).

Note--M. elliptica is similar to M. coreia, but it differs by the smaller flower, the exserted stamens and the curved filament. M. elliptica is confined only in 
the south, but $M$. coreia is widespread in the country but not in the south.

5. Morinda nana Craib, Bull. Misc. Inform., Kew 1932: 434. 1932 \& Fl. Siam. Enum. 2: 177. 1932. Type: Thailand, Nakhon Phanom, Ta Uten district, A.F.G. Kerr 8461 (holotype BK!; isotypes ABD!, BM!, K!).

Shrubs or scandent shrubs; young branches terete, bark smooth, greenish, with sparse and short hairs; older branches subterete, bark smooth, brownish to grey. Leaves variable, elliptic or fiddle-like shaped, $0.5-1$ by $1.5-2 \mathrm{~cm}$, coriaceous; base cuneate; margin entire; apex acute; upper leaf surface dark green, lower leaf surface greenish, with sparse and short hairs on both surfaces; lateral veins $4-5$ pairs, venation prominent; drying dark brown; petiole up to $3 \mathrm{~mm}$ long, glabrous. Stipules triangular, acute to acuminate, connate at base, usually persistent. Leafopposed inflorescence: capitula solitary, peduncle at anthesis sessile or up to $3 \mathrm{~mm}$ long. Flowers 5-6merous, fragrant, bisexual; calyx tube basally connate, with sparse and short hairs; calyx lobes obtuse or triangular; corolla hypocrateriform, white or greenish; corolla tube 6-7 mm long, glabrous or with sparse and short hairs on both sides; corolla lobes oblong, $1-2$ by $4-5 \mathrm{~mm}$ long, with sparse and short hairs on both sides. Stamens included or exserted in the corolla tube, attached to the tube by a short filament; filaments linear, 1-1.5 mm long; anthers yellow, oblong, 3$4 \mathrm{~mm}$ long; style white, 9-10 $\mathrm{mm}$ long, glabrous. Ovary 2-locular; stigma bilobed, $1.5-2 \mathrm{~mm}$, included or exserted in the corolla tube. Infructescences subglobose or oblique, with sparse and short hairs. Seed ellipsoid or ovoid, testa rough.

Thailand.- NORTH-EASTERN: Nakhon Phanom [Ta Uten district, 6 Feb. 1924, Kerr 8461 (ABD, BK, BM, K)]; EASTERN: Amnat Charoen [Chanuman district, Ban Bung Kheio, 26 Mar. 1923, Kesonbua 61 \& 62 (KKU)].

Distribution.-Endemic to Thailand.

Ecology.- Very common along stream banks, alt. ca. 200 m. Flowering: February-March.

Vernacular.- 'Yo phaya mai' (Eastern).

Note.-M. nana differs from the other Morinda and its allies by the smaller leaves and inflorescence.

6. Morinda pandurifolia Kuntze, Rev. Gén. 1: 289: 1891; Craib, Fl. Siam. Enum. 2: 178.1934. M. persicaefolia var. pandurifolia (Kuntze) Pitard in Lecomte, Fl. Indo-Chine 3: 122. 1922. Type: Cambodia, Campong luang, Thorel s.n. (isotype P!).M. pandurifolia Kuntze var. tenuifolia Craib, Fl. Siam. Enum. 2: 178. 1934. Type: Thailand, Surat Thani,
Ban Na, A.F.G. Kerr 18124 (holotype BK!, isotype ABD!). - M. longifolia Craib, Bull. Misc. Inform., Kew 1932: 434. 1932 \& Fl. Siam. Enum. 2: 177. 1934. Type: Thailand, Tak, Raheng, A.F.G. Kerr 4577 (holotype BK! isotypes ABD!, BM!), synon. nov.

Shrubs or scandent shrubs; young branches terete, bark smooth, greenish, with sparse and short hairs; older branches subterete, bark smooth, brownish to grey. Leaves elliptic, lanceolate, oblong or fiddle-like shaped, $1-3.5$ by $3-15 \mathrm{~cm}$, coriaceous; base cuneate; margin entire; apex acute or acuminate; upper leaf surface dark green, lower leaf surface greenish, with sparse and short hairs on both surfaces; lateral veins 8-12 pairs, venation prominent; drying dark brown; petiole $1-3 \mathrm{~cm}$ long, glabrous or with sparse and short hairs. Stipules triangular, acute to acuminate, connate at base, usually caducous. Leaf-opposed inflorescence: capitula solitary; peduncle at anthesis sessile or up to $4 \mathrm{~mm}$ long. Flowers 5-6-merous, fragrant, bisexual; heterostylous; calyx tube basally connate, with sparse and short hairs; calyx lobes obtuse or triangular; corolla hypocrateriform, white or greenish, corolla tube $1.5-1.8 \mathrm{~cm}$, inside glabrous or with sparse and short hairs, outside puberulous; corolla lobes oblong, $3-5.5$ by $15-14.5 \mathrm{~mm}$, inside glabrous, outside puberulous or with spares and short hairs. Stamens included or exserted in the corolla tube, attached to the tube by a short filament; filaments linear, 1$4 \mathrm{~mm}$ long; anthers yellow, oblong, 5.5-6.5 mm long; style white, 3-5 mm long in brevistylous flowers, 8$10 \mathrm{~mm}$ long in longistylous flower, glabrous. Ovary 2-locular; stigma bilobed, 1-2 mm long, included or exserted in the corolla tube. Infructescences globose, subglobose or oblique, with 23-50 fruitlets; fruitlet with sparse and short hairs, green. Seed ellipsoid or ovoid, $4.5-6.5$ by $7-11.5 \mathrm{~mm}$, testa rough.

Thailand.- NORTHERN: Tak [Raheng, Kerr 4577 (BK, ABD, BM]; NORTH-EASTERN: Loei [Wang Sapung district, 15 April 1991, Din 161 (BKF); Phu Kradueng, 15 April 1991, Sin 161 (BKF)]; Maha Sarakham [Kosum Phisai, May 1968, Smitinand 10444 (BKF, P)]; CENTRAL: Ang Thong [6 Mar. 1971, Maxwell 71-120 (AAU, BK); Wat Glang, Howa Pie, Muang district, 31 May 1975, Maxwell 75-570 (AAU, BK, L)].

Ecology.- In dry evergreen and deciduous dipterocarp forests, stream banks, alt. 0-150 m. Flowering period: December-April.

Distribution.- Indo-China.

Vernacular.— 'Yo pa' (Ang Thong).

Note.-M. pandurifolia is distinguished by its shrubs or scandent shrubs. The margin of leaf is entire and usually curving inwards in the middle part. 
7. Morinda scabrida Craib, Bull. Misc. Inform., Kew 1932: 435. 1932 \& Fl. Siam. Enum. 2: 179. 1934. Type: Thailand, Kanchanaburi, Bow Noi, A.F.G. Kerr 10260 (holotype BK!; isotypes ABD!, BM!, K!).

Shrubs up to $1 \mathrm{~m}$; young branches terete to subterete, bark smooth, greenish, with sparse and short hairs; older branches subterete, bark smooth or shallowly fissured, brownish to grey. Leaves elliptic, oblong or obovate, 6-12 by $17-28 \mathrm{~cm}$, coriaceous; base cuneate; margin entire; apex acute or acuminate; upper leaf surface dark green, with sparse and short hairs, lower leaf surface greenish, tomentose; lateral veins 6-10 pairs, venation prominent; drying pale green; petiole 1.5-2.6 cm long, with spares and short hairs. Stipules triangular, acute to acuminate, connate at base, usually persistent. Leaf-opposed inflorescence: capitula solitary or paired; peduncle at anthesis 1-3.5 cm long. Flowers 4-6-merous, fragrant, bisexual; heterostylous; calyx tube connate, with sparse and short hairs; calyx lobes obtuse; corolla infundibular, white, greenish or purple; corolla tube $2-3.2 \mathrm{~cm}$ long, inside glabrous, outside with sparse and short hairs; lobes ovate or elliptic, $4-8$ by $8-17 \mathrm{~mm}$. Stamens included in the corolla tube, attached to the tube by a short filament; filaments linear, 1-2 mm long; anthers yellow, oblong, 3-4 mm long; style white, 12 $15 \mathrm{~mm}$ long in brevistylous flower, $1.5-1.8 \mathrm{~cm}$ long in longistylous flower, glabrous. Ovary 2-locular; stigma bilobed, 2-4 $\mathrm{mm}$ long, included in corolla tube. Infructescences oblique, with 11-50 fruitlets, ripening black. Seed ellipsoid or ovoid, 3-5 by 5$7 \mathrm{~mm}$, testa rough.

Thailand.- SOUTH-WESTERN: Kanchanaburi [Sangkhlaburi district, 9 Feb. 1960, BKF 20 (BKF, K, L), 14 June 2003, Kesonbua 81 (KKU), 31 Mar. 1967, Nimanong 3 (BKF), 24 Mar. 1967, Prayad 713 (BK, BKF); Bow Noi, 18 Jan. 1926, Kerr 10260 (ABD, BK, BM, K); Thong Pha Phum district, 15 June 2003, Kesonbua 82 (KKU)].

Distribution.-Endemic to Thailand.

Ecology.- In hill evergreen and mixed deciduous forests, alt. 250-700 m. Flowering period: JanuaryMarch.

Note.- M. scabrida is closely similar to M. angustifolia, but it differs by having fused fruitlets and the leaf drying pale green, with short hairs on the upper surface and dense hairs on the lower one.

8. Morinda talmyi Pierre ex Pitard in Lecomte, Fl. Indo-Chine 3: 426. 1922; Craib, Fl. Siam. Enum. 2: 179. 1932. - M. persicaefolia Ham. var. talmyi Pitard in Lecomte, Fl. Indo-Chine 3: 426. 1922. Type:

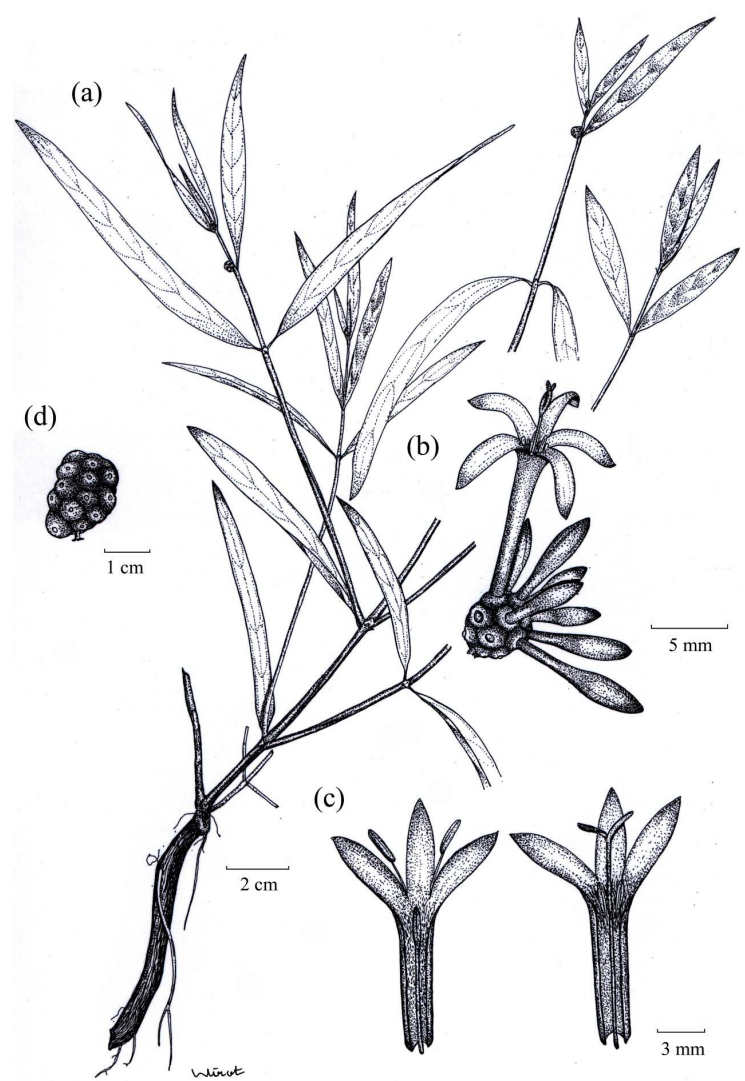

Fig. 2 M. talmyi Pierre ex Pitard: (a) habit; (b) inflorescence; (c) longitudinal section of brevistylous and longistylous flowers; (d) infructescence. (a) and (d) from W. Kesonbua 71; (b) from W. Kesonbua 72; (c) from W. Kesonbua 71 (brevistylous flower) and W. Kesonbua 72 (longistylous flower). Drawn by W. Kesonbua.

Cambodia, Pierre 3217 (lectotype P!, selected here). Fig. 2.

Small shrubs or scandent shrubs; young branches subterete, bark smooth, greenish, glabrous; older branches subterete, bark smooth, brownish to grey. Leaves elliptic, lanceolate, oblong or fiddle-like shaped, $1-3.5$ by $3-11.5 \mathrm{~cm}$, membranaceous; base cuneate; margin entire; apex acute or acuminate; upper leaf surface light green, lower leaf surface greenish, glabrous on both surfaces; lateral veins 58 pairs, venation obscure; drying dark brown; petiole 2-7 mm long, glabrous. Stipules triangular, acute to acuminate, connate at base, usually persistent. Leafopposed inflorescence: capitula solitary; peduncle at anthesis sessile or up to $2 \mathrm{~mm}$ long. Flowers 5-6-merous, fragrant, bisexual; heterostylous; calyx tube basally connate, glabrous; calyx lobes obtuse or triangular; corolla hypocrateriform, white or greenish; corolla tube $0.7-1 \mathrm{~cm}$ long, inside with sparse and 
short hairs, outside glabrous; corolla lobes oblong, $1.5-2.5$ by $5.5-7 \mathrm{~mm}$. Stamens included or exserted in the corolla tube, attached to the tube by a short filament; filaments linear, 1-4 mm long; anthers white or yellowish grey, oblong, 2-3 mm long; style white, 4-7 mm long in brevistylous flower, $8-12 \mathrm{~mm}$ long in longistylous flower, glabrous. Ovary 2-locular; stigma bilobed, 2-3 mm long, included or exserted in the corolla tube. Infructescences subglobose or oblique, glabrous, with 15-35 fruitlets, greenish. Seed ellipsoid or ovoid, 3-5 by 5-7 $\mathrm{mm}$, testa rough.

Thailand.- NORTH-EASTERN: Nong Khai [Phon Phisai district, 6 June 1963, Chantaramuck 472 (BK)]; Nakhon Phanom [Ban Teng, 24 Feb. 1993, Chantaranothai, Parnell, Middleton \& Simpson 888 (TCD)]; EASTERN: Chaiyaphum [Ban Lad Yai, 9 Jan. 1985, Paisooksantivatana Y 1522-85 (BK)]; Nakhon Ratchasima [Tha Chang, 16 May 1931, Kerr 20470 (ABD, BK, BM, TCD); Phimai district, 16 Mar. 1958, Smitinand 4474 (BKF), 16 Mar. 1930, TSBS 2156 (BKF)]; Si Sa Ket [Kantharalak district, 7 April 1966, Sangkhachand 231 (BK); Kanthararom district, 15 Mar. 1959, Suvarnakoses \& Smitinand 1549 (BKF, L, K, P)]; SOUTH- EASTERN: Nakhon Phanom [Tha Uthen district, 8 April 2002, Kesonbua $71($ KKU) \& 72 (KKU)]; Maha Sarakham [Borabue district, 29 Oct. 1965, Sutheesorn 732 (BK)].

Distribution.- Indo-China.

Ecology.- In dry evergreen and deciduous dipterocarp forests, streamsides, alt. $200 \mathrm{~m}$. Flowering period: January-June.

Note.- M. talmyi is highly variable in size and shape of the leaves. The leaves are usually linear and only $1 \mathrm{~cm}$ wide, some specimens are oblong, elliptic or ovate and up to $3 \mathrm{~cm}$ wide. It is similar to $M$. pandurifolia, but differs by its glabrous leaves and inflorescences.

9. Morinda tomentosa Heyne ex Roth, Nov. Pl. Sp.: 147. 1821; Kurz, Forest Fl. Burma 2: 60. 1877; Craib, Fl. Siam. Enum. 2: 178. 1934. Type: Wight 1321 (isotype K!). - M. tinctoria Roxb. var. tomentosa Hook. f., Fl. Brit. Ind. 3: 156.1880 ; Pitard in Lecomte, Fl. Indo-Chine 3: 425. 1922. Type: India, not seen.-M. pumila Craib, Bull. Misc. Inform., Kew 1932: 434. 1932 \& Fl. Siam. Enum. 2: 177. 1934. Type: Thailand, Chaiyaphum, Pak Pang, A.F.G Kerr 19981 (holotype BK!; isotypes ABD!, K!, BM!), synon. nov. Fig. 3.

Trees up to $15 \mathrm{~m}$; young branches terete, bark smooth, greenish, densely puberulous; older branches subterete, bark shallowly fissured, brownish to grey, with sparse and short hairs. Leaves elliptic, 8-9.5

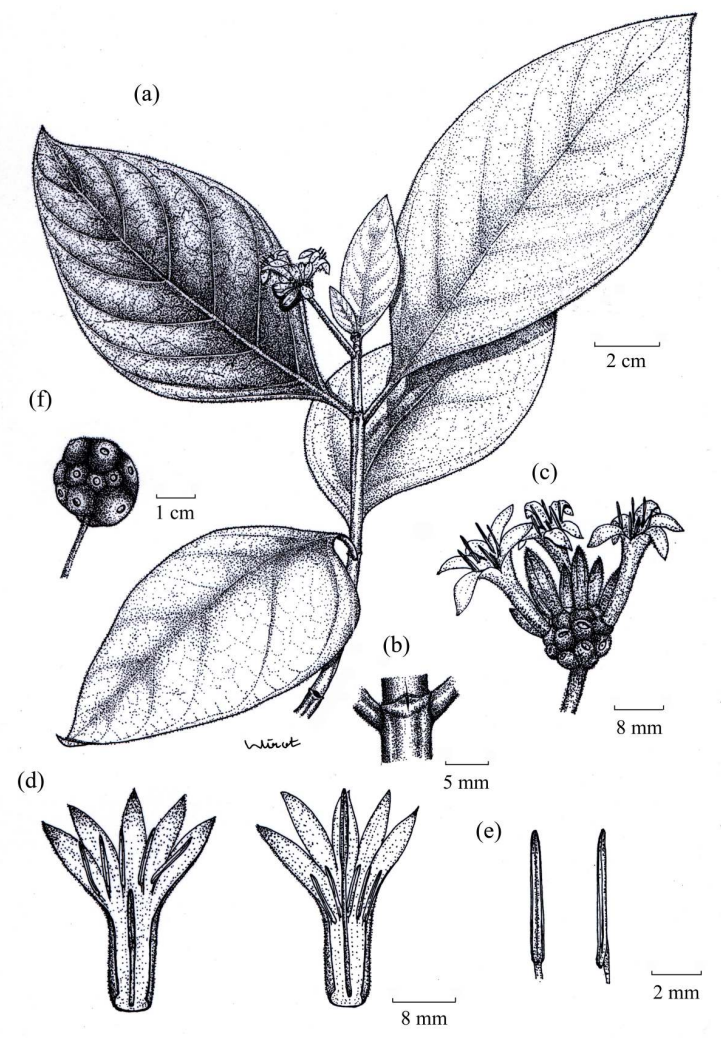

Fig. 3 M. tomentosa Heyne ex Roth: (a) habit; (b) stipule; (c) inflorescence; (d) longitudinal section of brevistylous and longistylous flowers; (e) anthers; (f) infructescence. (a), (b), (c), (e), and (f) from W. Kesonbua 22; (d) from W. Kesonbua 22 (brevistylous flower) and W. Kesonbua 35 (longistylous flower). Drawn by W. Kesonbua.

by $15.5-23 \mathrm{~cm}$, coriaceous; base cuneate; margin entire; apex acute or acuminate; upper leaf surface dark or light green, puberulous, lower leaf surface greenish, tomentose; lateral veins $8-12$ pairs, venation prominent, domatia usually present in axils of secondary vein on the lower surface; drying dark black; petiole 1-3 cm long, with dense hairs. Stipules triangular, acute to acuminate, connate at base, usually caducous. Leaf-opposed inflorescence: capitula solitary or paired; peduncle at anthesis $2-4.5 \mathrm{~cm}$ long. Flowers 5-6-merous, fragrant, bisexual; heterostylous; calyx tube basally connate, puberulous; lobes truncate or denticulate; corolla hypocrateriform, white or greenish; corolla tube $1.5-1.8 \mathrm{~cm}$ long, inside glabrous, outside puberulous; corolla lobes oblong, 35.5 by $10-14.5 \mathrm{~mm}$. Stamens included or exserted in the corolla tube, attached to the tube by a short filament; filament linear, straight, 1-4 mm long; anthers yellow, oblong, 5.5-6.5 mm long; style white, 
glabrous, 1.2-1.5 cm long in brevistylous flower, 1.5$1.8 \mathrm{~cm}$ long in longistylous flower. Ovary 2-locular; stigma bilobed, included or exserted in the corolla tube. Infructescences globose or subglobose, with 15-25 fruitlets, fruitlet with short and dense hairs, ripening black. Seed ellipsoid or ovoid, $4.5-6.5$ by 7-11.5 mm long, testa rough.

Thailand.- NORTHERN: Mae Hong Son [Ban Sop Pong, 12 June 1989, Anderson 6023 (CMU)]; Chiang Mai [not located, 28 July 1992, Larsen, Larsen, Niyomdham, Sirirugsa, Tarvengadum \& Nrgaard 43719 (P); Doi Inthanon, 23 April 1989, Maxwell 89-505 (BKF, CMU, L), 16 July 1988, Phengklai et al. 6544 (AAU, BKF, E), Maxwell 7 (BKF), 22 Aug. 1987, Maxwell 87-860 (BKF, CMU, L), 7 April 1988 (K); Doi Muang Awn, Sankampang district, 21 June 1990, Maxwell 90-659 (CMU, E, L), 25 June 1992, Maxwell 92-321 (CMU, E, L, P); Doi Suthep, 23 April 1991, Dawson 24 (BKF), 16 July 1988, Fukuoka T-62007 (BKF, L), 19 Sept. 1996, Maxwell 88-442 (AAU, BK, BKF, CMU, L)]; Chiang Rai [Khun Tan, 20 April 1942, Sman 132 (BKF), 15 April 1989, Chalerm 141 (BKF)]; NORTH-EASTERN: Loei [Wang Sapung, 17 April 1989 Din 32 (BKF), 16 Jan. 1990, Din 130 (BKF), 27 April 1991, Din 273 (BKF), 25 May 1934, Pan 105 (BKF); Phu Kradueng, 27 April 1998, Fukuoka T-63657 (BKF, L)]; Khon Kaen [Khon Kaen University, 2 Mar. 2001, Kesonbua 4 (KKU), 26 Mar. 2001, Kesonbua 9 (KKU), 5 May 2001, Kesonbua 22 (KKU), 13 May 2001, Kesonbua 35 (KKU)]; EASTERN: Chaiyaphum [Pak Pang, Kerr 19981 (ABD, BK, BM, K]; SOUTH-WESTERN: Kanchanaburi [Erawan National Park, 19 April 1968, van Beusekom \& Phengklai 512 (P)]; SOUTH-EASTERN: Chon Buri [Khao Khieo, 20 June 1975, Maxwell 75-622 (AAU, BK, L), 26 June 1976, Maxwell 76-408 (AAU, BKF, L)].

Distribution.- India, Myanmar, Indo-China.

Ecology.- In dry evergreen and deciduous dipterocarp forests, alt. $100-350 \mathrm{~m}$. Flowering period: February-May.

Vernacular.— 'Kho khamin', 'yo pa', 'sa kae', 'sa koei', 'hatsa koei' (Northern); 'talum phuk' (Khon Kaen).

Note.- M. tomentosa is very closely similar to M. coreia, but it differs by having hairs on the leaf surface which is variable, both surfaces and usually having dense hairs, some specimens have only sparsely distributed hairs.

Acknowledgements: We thank the Biodiversity Research and Training Program (BRT T_145031), the Faculty of
Science, Ubon Ratchathani University and the Department of Biology, Faculty of Science, Khon Kaen University for supporting this study. We also thank the directors and curators of AAU, ABD, BK, BKF, BM, CMU, E, K, KKU, L, P, PSU, QBG, TCD, and Herbarium of Biology Department, Chiang Mai University, for making the specimens available for this study. The reviewers are thanked for their valuable comments.

\section{REFERENCES}

1. Linnaeus C (1753) Species plantarum 1. L. Salvius, Holmiae.

2. Razafimandimbison SG, Bremer B (2011) Nomenclatural changes and taxonomic notes in the tribe Morindeae (Rubiaceae). Adansonia 33, 283-309.

3. Razafimandimbison SG, Ekman S, McDowell TD, Bremer B (2012) Evolution of growth habit, inflorescence architecture, flower size, and fruit type in Rubiaceae: its ecological and evolutionary implications. PLOS ONE 7, e40851.

4. Igersheim A, Robbrecht E (1993) The character states and relationships of the Prismatomerideae (RubiaceaeRubiodeae)—comparisons with Morinda and comments on the circumscription of the Morindeae s. str. Opera Bot Belg 6, 61-79.

5. Baillon HE (1879) Sur l'organisation et les limites du genre Morinda. Bull Mens Soc Linn Paris 1, 217-8.

6. Vercourt B (1958) Remarks on the classification of the Rubiaceae. Bull Jard Bot État Brux 28, 209-81.

7. Bremekamp CEB (1966) Remark on the position, the delimitation and subdivision of the Rubiaceae. Acta Bot Neerl 15, 1-33.

8. Johansson JT (1987) Pollen morphology of the tribe Morindeae (Rubiaceae). Grana 26, 134-50.

9. Johansson JT (1994) The genus Morinda (Morindeae, Rubioideae, Rubiaceae) in New Caledonia: taxonomy and phylogeny. Opera Bot 122, 1-67.

10. Robbrecht E (1988) Tropical woody Rubiaceae. Characteristic features and progressions. Contributions to a new subfamilial classification. Opera Bot Belg 1, $1-271$.

11. Razafimandimbison SG, Rydin C, Bremer B (2008) Evolution and trends in the Psychotrieae alliance (Rubiaceae)—A rarely reported evolutionary change of many-seeded carpels from one-seeded carpels. $\mathrm{Mol}$ Phylogenet Evol 48, 207-23.

12. Razafimandimbison SG, McDowell TD, Halford DA, Bremer B (2009) Molecular phylogenetics and generic assessment in the tribe Morindeae (RubiaceaeRubioideae): How to circumscribe Morinda L. to be monophyletic? Mol Phylogenet Evol 52, 879-86.

13. Craib WG (1932) Contribution to the Flora of Siam XXXVI. Bull Misc Inform 9, 425-37.

14. Craib WG (1934) Florae Siamensis Enumeratio II. Siam Society, Bangkok. 